\title{
REVIEW
}

\section{Calreticulin and cellular adhesion/migration-specific signalling pathways}

\author{
Eva Szabo, Sylvia Papp, Michal Opas \\ Department of Laboratory Medicine \& Pathobiology, University of Toronto, Toronto, Ontario, Canada
}

Received $15^{\text {th }}$ July 2005.

Published online $1^{\text {st }}$ October 2005.

\begin{abstract}
Summary
Calreticulin is a $\mathrm{Ca}^{2+}$-binding protein of the ER/SR, from where it acts as a chaperone, and affects calcium homeostasis, gene expression and cell adhesion. Cell adhesion to the extracellular matrix can generate transmembrane signals important for cell survival and migration. In a variety of cell types, integrin stimulation by ECM proteins, such as fibronectin, leads to changes in intracellular protein tyrosine phosphorylation. Tyrosine phosphorylation leads to the co-localization of focal adhesion kinase, vinculin and paxillin at focal contacts. Interaction between focal adhesion kinase and paxillin is critical for the activation of signaling cascades involved in cell survival and motility. Fibroblasts either over- or underexpressing calreticulin show differences in their adhesive properties, which are related to the calmodulin/CaMKII pathway. Inhibition of these pathways causes the weekly adhesive calreticulin underexpressing cells to behave like the calreticulin overexpressers, through increased spreading and increased levels of focal adhesion kinase, paxillin and fibronectin. We propose that calreticulin, via its $\mathrm{Ca}^{2+}$-homeostatic effects, may affect fibronectin synthesis and matrix assembly by modulating fibronectin gene expression, and by influencing formation of cellular adhesions, both of which are instrumental in matrix assembly and remodelling. Interestingly, it appears that besides the calmodulin/CaMKII pathway, differential calreticulin expression also modulates the c-src pathway.
\end{abstract}

Keywords: adhesion - motility - calreticulin - src - calmodulin - CamK II

\section{INTRODUCTION}

Many studies have indicated that calreticulin is an endoplasmic reticulum (ER)-resident protein, albeit the protein has been found in various subcellular

Eva Szabo, Department of Laboratory Medicine \& Pathobiology, University of Toronto, 1 King's College Circle, Medical Sciences Building, Rm. 6326, Toronto, Ontario, M5S 1A8 Canada

葛 eva.szabo@utoronto.ca

용 + (416) 978-8947

且 + (416) 978-5959 and extracellular loci (Papp and Opas 2003). One of the more intriguing functions of calreticulin is its control of cell adhesiveness (Papp et al. 2003). The proteins involved in cell adhesions are either part of 1) the subplasmalemmal cytoskeleton (focal adhesion proteins such as paxillin, focal adhesionkinase (FAK) and vinculin), 2) the cytosol (signalling molecules such calmodulin and CaMK II), or 3) the extracellular matrix (fibronectin). Thus, for calreticulin to affect these protein levels, there must be an ER signalling pathway involved.

The signalling mechanism might sense the levels of calreticulin in the ER and convey that information through a signaling pathway to the nucleus to up-regulate or attenuate gene transcription (Fig. 1). There are a number of 
signalling pathways, such as the calmodulin/CaMK II, Src and ERK pathways, that may be activated either in parallel or separately, to modulate focal adhesions (Fig. 2). It has been shown that overexpression of calreticulin leads to the formation of more prominent focal contacts and this is in part due to upregulation of vinculin and decreased tyrosine phosphorylation (Fadel et al. 1999, Opas et al. 1996).

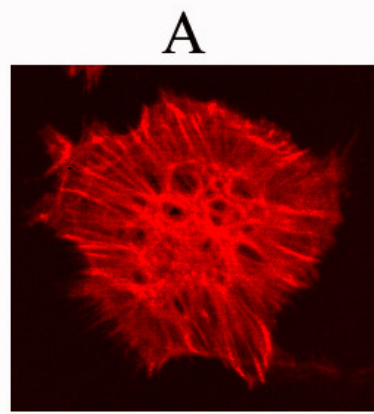

actin

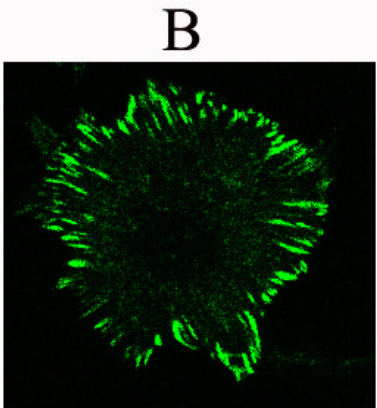

vinculin

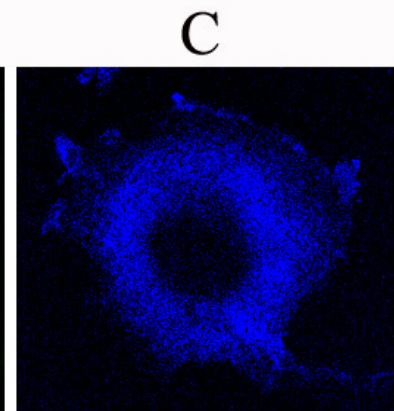

calreticulin

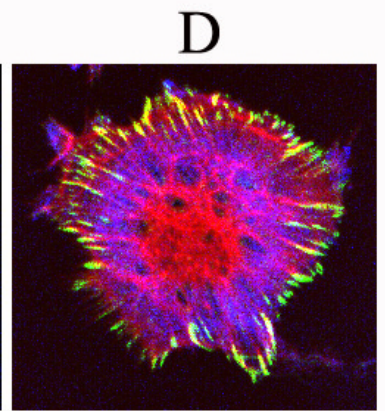

merge

Fig. 1. Distribution of adhesion-related cytoskeletal proteins and calreticulin. Calreticulin-overexpressing fibroblasts were triple labelled to visualize actin (a), vinculin (b) and calreticulin (c). It is evident that cytosolically distributed adhesionrelated cytoskeleton (actin stress fibres) and subplasmalemmal adhesion-related cytoskeleton (vinculin-rich focal contacts) co-localize to a large extent. This is in contrast to the calreticulin-rich ER, which only slightly co-localizes with either actin or vinculin in certain areas. All three labels have been merged in (d) to show the degree of co-localization of the labels.

\section{ROLE OF CALRETICULIN IN FOCAL ADHESION BASED CELL ADHESIVENESS}

The ability of cells to form focal contacts and adhere to their underlying substratum is critical for motility, differentiation and survival. The formation of focal contacts is carefully regulated by cells and is affected by a number of signalling pathways, some being calcium dependent. The ER resident protein, calreticulin, has been implicated in the control of cell to cell and cell to substratum adhesions, through its calcium related functions.

Calreticulin is a soluble protein found within the lumen of the ER. It has three domains, the compact globular $\mathrm{N}$-domain which does not bind calcium, the extended arm formed by the hairpin loop of the P-domain, which binds calcium with low capacity, high affinity and finally the carboxy terminal $\mathrm{C}$ domain which binds calcium with high capacity, low affinity (Parodi et al. 2000).

The primary function ascribed to calreticulin has been as a chaperone protein, where it binds to newly synthesized glycoproteins, preventing aggregation and allowing the proteins to attain their correct folding conformation (Helenius et al. 1997). In addition to its calcium storage function, calreticulin also has an important role in the regulation of calcium homeostasis (Michalak et al. 2002). Calcium regulation is an important part of the adhesion mechanism (Burridge and Chrzanowska-Wodnicka 1996, Sastry and Burridge 2000). Alterations in the level of expression of calreticulin indeed affect cellular adhesion and motility (Opas et al. 1996, Fadel et al. 1999, 2001). Calreticulin deficient cells have impaired adhesion (Liu et al. 1994, Coppolino et al. 1997). It has been suggested that this may be mediated by direct interaction between calreticulin and KxGFFKR sequence of $\alpha$-integrins (Dedhar 1994, Coppolino et al. 1995). Consequently, to functionally affect integrins clustered in focal contacts, calreticulin should be present in the cytoplasm, but there is no strong direct evidence for this. Thus, calreticulinmodulated changes in cell adhesiveness have to be correlated with upregulation of adhesion specific proteins.

Overexpression of calreticulin increases both cell to substratum and cell-to-cell adhesiveness, and establishes vinculin rich cell-to-cell junctions by increasing overall vinculin levels in cells (Opas et al. 1996). Thus, the adhesion related effects of differential calreticulin expression are vinculin 
mediated and the absence of calreticulin from focal contacts or the cytoplasm indicates that, in vivo, the adhesion-related functions are performed from within the ER lumen (Fig. 1).

In addition, overexpressed and cytoplasmically targeted GFP-calreticulin did not localize to focal contacts. Furthermore, targeting of calreticulin to the cytoplasm either by microinjection or by the expression of a leaderless calreticulin had no effect on cell morphology, the actin cytoskeleton or cell adhesion. Most importantly, studies show that both transcriptional activation by steroid receptors and cell adhesion in vivo are affected only by the full length, ER-targeted form of calreticulin, but not by a truncated, cytosolically targeted mutant protein (Opas et al. 1996, Michalak et al. 1996, Fadel et al. 1999).

More recently it has been suggested that calreticulin may be involved in an integrindependent calcium-signalling pathway, rather than direct regulation of integrin activity (Coppolino et al. 1997). While this observation requires further investigation, it is not inconsistent with the hypothesis that calreticulin may function in adhesion regulation as a "signalling" molecule from within the ER lumen (Michalak et al. 2002, Papp et al. 2003) (Fig. 2).

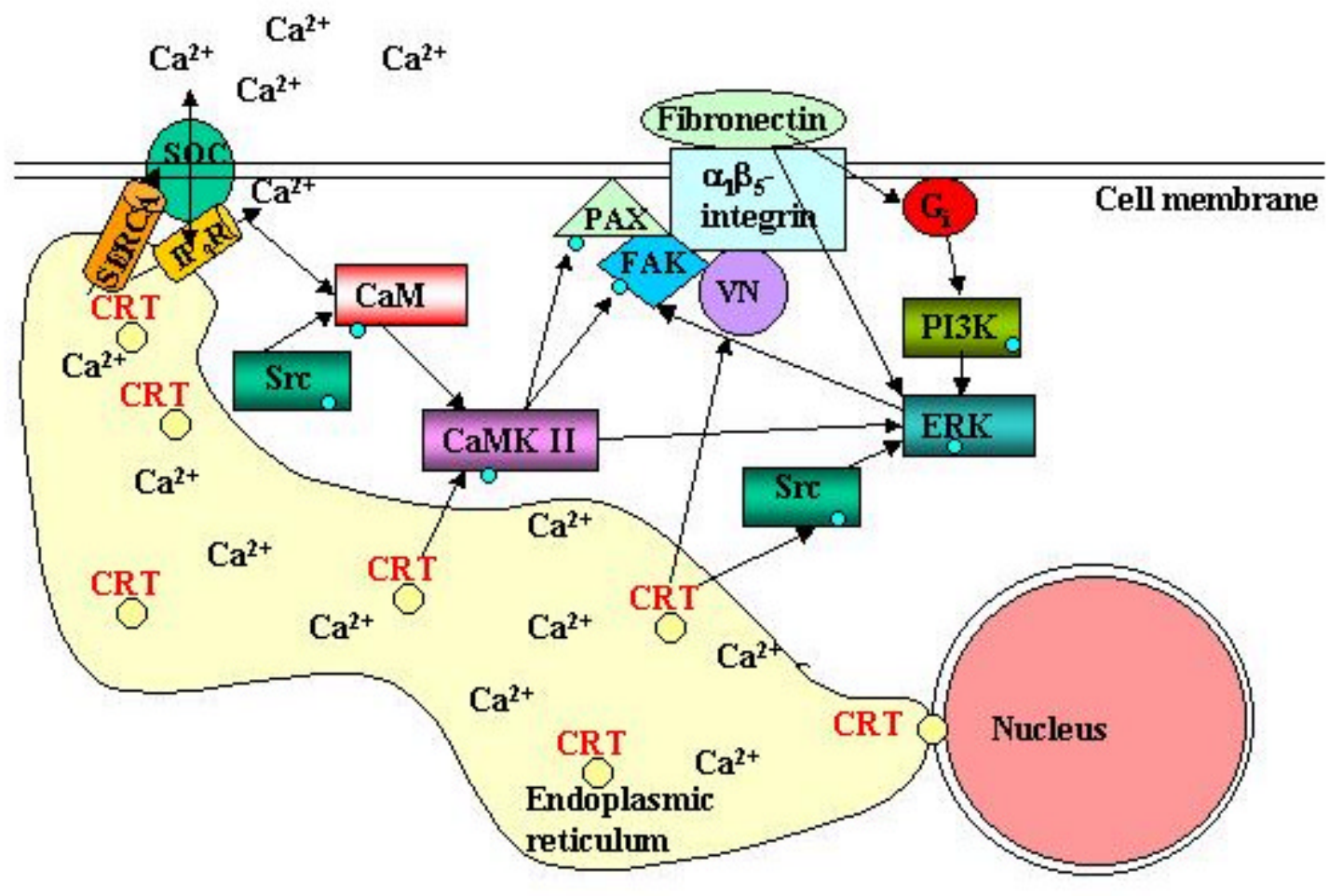

- phosphorylation

Fig.2. Hypothetical involvement of calreticulin in a number of signalling cascades. Calreticulin through its role in calcium homeostasis is indirectly affecting a number of signalling cascades.

\section{CALRETICULIN EXPRESSION AND FOCAL ADHESION PROTEINS}

Focal adhesions contain numerous structural proteins, such as actin, $\alpha$-actinin and vinculin, as well as, many regulatory proteins (Sastry and Burridge 2000), such as FAK, paxillin, tensin and zyxin (Otey 1996, Burridge et al. 1992, Crawford et al. 1992). The regulatory proteins are either phosphorylated or are substrates for tyrosine kinases in an adhesion-dependent manner (Burridge and Chrzanowska-Wodnicka 1996, Ilic et al. 1997,). In addition, regulatory proteins such as Src, protein kinase $\mathrm{C}$, calmodulin and CaMK II, which are part of major signalling cascades, are also involved with adhesion formation (Fig. 2).

Furthermore, the clustering and immobilization of integrins at focal contacts appears to participate 
in cell signalling via tyrosine phosphorylation events (Juliano and Huskill 1993) and tyrosine phosphorylation is implicated in focal adhesion assembly (Kornberg and Juliano 1992). Overexpression of calreticulin also increases $\mathrm{N}$ cadherin levels and decreases tyrosine phosphorylation of cellular proteins, such as $\beta$ catenin (Fadel et al. 2001). $\beta$-catenin is a component of the cadherin-mediated adhesion complexes and is also part of the Wnt signalling pathway (Hutzfeld 1999). Calreticulin from the ER influences tyrosine phosphorylation of $\beta$-catenin but not its expression: for example, $\beta$-catenin is underphosphorylated in calreticulin overexpressing cells, but protein and mRNA levels remain the same in these cells (Fadel et al. 2001).

Calreticulin can also affect cell adhesion through the calmodulin and CaMK II pathway. In calreticulin underexpressing cells, the calmodulin levels as well as active CaMK II levels, are higher than in their overexpressing counterparts. Inhibition of calmodulin or CaMK II rescues the calreticulin underexpresser phenotype, through increased cell spreading and increased paxillin and FAK phosphorylation and protein levels (Szabo unpublished data). FAK binds to paxillin and in turn paxillin provides docking sites for $\mathrm{Src}$ and vinculin, targeting them to the focal contacts (Burridge and Chrzanowska-Wodnicka 1996, Brown et al. 1998). Additionally, in calreticulin underexpressing cells, c-src phosphorylation at tyrosine 418 (indicating c-src activation) is higher compared with the calreticulin overexpressing cells (Papp, unpublished data). Src has been shown to phosphorylate calmodulin at tyrosine 99, which increases the affinity of calmodulin for CaMK II (Sacks et al. 1989). Also, the activated Src as well as calmodulin and CaMK II have been show to activate the ERK pathway independent of each other (Belcheva et al. 2005, Illario et al. 2005). The ERK pathway in turn plays an important role in upregulating paxillin and FAK, whereby ERK phosphorylates paxillin and FAK, recruiting them to focal contacts (Ishibe et al. 2004). In calreticulin underexpressing cells, calmodulin levels are upregulated, thus increasing the activity of CaMK II, which in turn could activate the ERK pathway causing an increase in paxillin levels. In calreticulin-null cells, ERK 1 expression is higher than in wild type cells, but ERK 2 expression seems to be unaffected (Michalak, unpublished data).

The ERK pathway is also upregulated by the binding of fibronectin to integrins (Illario et al 2005). However, in calreticulin underexpressing cells, fibronectin levels are lower than in overexpressing cells, therefore activation of the ERK pathway would be thought to be downregulated. The decreased fibronectin deposition and lower vinculin levels in calreticulin underexpressers could also account for the reduced focal adhesion properties of these cells compared with the overexpressers, since vinculin and fibronectin regulate the recruitment and assembly of the focal adhesion proteins FAK and paxillin at the site of integrins. This indicates that differential calreticulin levels regulate fibronectin expression. Overexpression of calreticulin leads to the induction of the fibronectin gene and increased fibronectin deposition, explaining the increased spreading observed when calreticulin is overexpressed. When the calmodulin/CaMK II pathway is inhibited, fibronectin protein levels and deposition increase and the calreticulin underexpressing cells take on the morphology of calreticulin overexpressers (Szabo, unpublished data).

When calreticulin underexpressing cells are plated on fibronectin coated substrata, cell spreading is induced. Calreticulin underexpressers overcome their poorly adhesive phenotype by induction of many tensin-rich fibrillar adhesions, thus compensating for the low levels of vinculin in these cells. The calreticulin overexpressing cells form many vinculin-rich focal contacts as opposed to tensin-rich adhesions, since vinculin levels are elevated in these cells. (Papp et al., submitted).

Another indirect way that calreticulin may influence cell adhesion is through its effects on the transmembrane influx of calcium via store operated calcium channels (SOCs), conceivably by controlling $\mathrm{InsP}_{3}$-releasable endoplasmic reticulum calcium stores (Fasolato et al. 1998, Mery et al. 1996, Bastianutto et al. 1995, Xu et al. 2000). Calreticulin also affects the function of SERCA2b and the $\mathrm{InsP}_{3} \mathrm{R}$ (Camacho and Lechleiter 1995, Jouaville et al. 1999), both of which may be structurally coupled to SOCs (Lockwich et al. 2001). The structural relationship between the ER/SR calcium release channels and SOCs of the plasma membrane has been a matter of controversy, however, substantial evidence points to structural coupling between the two channels (Putney 1999, 2005). In view of the heterogeneity of the ER/SR (Meldolesi and Pozzan 1998, Petersen et al. 2001), it is intuitive that not all of the ER is coupled to the plasma membrane, however morphological data supporting this notion are scarce.

Trp (a putative SOC component) reportedly associates with $\operatorname{InsP}_{3} \mathrm{R}$, SERCA and caveolin in caveolar calcium signalling complexes (Lockwich et al. 2000, Lockwich et al. 2001). Calreticulin was shown to co-immunoprecipitate with caveolin (Darby et al. 2000). Caveolin co-clusters with $\alpha_{1}$ integrins and its downregulation inhibits $\alpha_{1}$ integrin-mediated adhesion to fibronectin (Wei et al. 1999). Furthermore, tyrosine phosphorylated caveolin has been localized to focal contacts (Volonte et al. 2001). Finally, in fibroblasts, the $\mathrm{InsP}_{3} \mathrm{R}$ was localized to focal contacts (Sugiyama et al. 2000). 


\section{EXTRACELLULAR CALRETICULIN AND CELL ADHESION}

Extracellular calreticulin is involved in cellular adhesion and migration but its role is unclear, since it does not possess a transmembrane domain. Extracellular calreticulin is a $\mathrm{Clq}$ co-receptor (Ghiran et al. 2003, McGreal et al. 2001) which complexes with CD91 on phagocytes for apoptotic cell ingestion (Basu et al. 2001, Ogden et al. 2001, Vandivier et al. 2002), has antithrombotic effects (Kuwabara et al. 1995, Dai et al. 1997), inhibits melanoma cell spreading (White et al. 1995, Zhu et al. 1997) and inhibits angiogenesis (Pike et al. 1998, Pike et al. 1999), however the mechanisms behind these phenomena are unknown.

It was recently shown that thrombospondin mediates the disassembly of focal contacts by interacting with cell surface calreticulin (Goicoechea et al. 2000). The thrombospondinbinding site was mapped to the N-domain of calreticulin (Goicoechea et al. 2002) and biochemical evidence indicates the presence of a calreticulin complex with low-density lipoprotein receptor-related protein at the cell surface (Orr et al. 2003). Thrombospondin stimulates focal adhesion disassembly and motility through the heparin binding domain, hep I, which binds to calreticulin and leads to phosphoinositide 3-kinase (PI3K) activation, and stimulation of ERK and $\mathrm{G}_{\mathrm{i}}$-protein systems (Orr et al. 2002, Orr et al. 2003).

The sources of extracellular calreticulin have been subject to speculation, nevertheless the serum level of calreticulin in patients with systemic lupus erythematosus averages $4.44 \mu \mathrm{g} / \mathrm{ml}$ versus $0.42 \mu \mathrm{g} / \mathrm{ml}$ in control sera (Eggleton et al. 1997).

\section{CALRETICULIN AND CELL MOTILITY}

Calreticulin from within the lumen of the ER can also affect cell migration. It was indicated above that calreticulin affects cellular adhesion and focal adhesion protein assembly at the site of focal contacts, through its influence on a number of signalling pathways, such as Src, ERK and calmodulin/CaMK II (Fig. 2). The same pathways that are involved in adhesion to the underlying substratum are also affecting cellular migration. It was indicated earlier that the ERK pathway is indirectly affected by calreticulin, whereby ERK1 is upregulated in calreticulin-null cells (Michalak, unpublished data).

A number of studies indicate that the ERK pathway plays a crucial role in cell migration by phosphorylating paxillin and FAK, which are important parts of focal adhesions. Through the ERK pathway, MAPK-activated protein kinase 2/3 is activated, which has been shown to play an important role in directional migration (Huang et al. 2004). Thus, it can be hypothesized that calreticulin plays a more upstream role in this ERK pathway in relation to cellular migration, since it has been noted that calreticulin underexpressing cells migrate slower during wound closing than the overexpressers, but actual directionality of the migration is not affected (Szabo, unpublished data).

Other pathway that may regulate cellular migration are: the calmodulin/calcineurin and calmodulin/CaMK II pathways. These pathways have also been shown to play a crucial role in cell adhesion. When wound closing experiments were conducted, the importance of these pathways also became apparent. Inhibition of calmodulin with W7 increased single cell motility, in both calreticulin under- and overexpressing cells, but the wound closing experiments indicated a slight decrease in their ability to close the wound. When the cells were examined more closely, random cellular motility was increased, but directionality was lost. Thus, the cells tried to move in one direction and then retracted and tried moving in another direction unsuccessfully (Szabo, unpublished data).

Another study indicated that calcineurin, which is a downstream target of calmodulin (Fig. 2), determines directionality by sensing differential cytosolic calcium gradients. The same study also provided evidence that there is cross talk between CaMKII, another downstream target of calmodulin (Fig. 2), and calcineurin during directional migration (Wen et al. 2004). Since calreticulin has been implicated in controlling cytosolic calcium levels and calreticulin overexpressing cells have a higher cytosolic calcium concentration than calreticulin underexpressers, it can be deduced that migration and directionality can be indirectly affected by calreticulin (Opas et al. 1996, Fadel et al. 1999).

The inhibition of CaMK II with KN-62 not only affected adhesion as evidenced by increased cell spreading (Bouvard et al. 1998), but also influenced cell migration. Inhibition of CaMK II lead to increased cellular migration during wound closing in both calreticulin under- and overexpressing cells. The magnitude of the increase in cellular migration during wound closing was the most apparent when calreticulin was overexpressed and it was less apparent when calreticulin was underexpressed, suggesting a role for calreticulin in regulating this pathway (Szabo, unpublished data). Single cell motility increased in both calreticulin under- and overexpressing cells with KN-62 treatment. Unlike inhibition of calmodulin, CaMK II inhibition showed no visible effects on directionality of migration (Szabo, unpublished data), suggesting that this pathway is involved with cell motility and less so with directionality of cell migration. 
The three different pathways; ERK, calmodulin/calcineurin, and calmodulin/CaMK II, have been shown to affect migration or directionality and cross talk between the pathways leads to proper assembly and disassembly of focal contacts during cellular migration. Where does calreticulin fit in? Calreticulin offers a connection between these pathways, since it has been shown that it affects cellular adhesion and cell migration, whereby calreticulin down-regulation decreases adhesion and migration, whereas upregulation has the opposite effect.

\section{CONCLUDING REMARKS}

Although the mechanisms of calreticulin action on cell adhesion are still elusive, it is conceivable that the effects observed on focal adhesions may be due to the effects of calreticulin on multiple signalling pathways. These effects include changes to the cadherin/vinculin system, changes in tyrosine kinases and phosphatases, interaction with the Wnt pathway, direct effect on the calmodulin/CaMK II pathway, and interaction with $\mathrm{InsP}_{3}$ receptormediated signaling and steroid receptors on the cell surface. In addition, the effects of calreticulin expression on cell migration could be attributed to its differential regulation of the above-mentioned signalling cascades. More detailed studies of these pathways during cellular migration would lead to better understanding of the role of calreticulin during these events.

\section{ACKNOWLEDGEMENTS}

Sylvia Papp is a recipient of a Canada Graduate Scholarship from the Canadian Institutes of Health Research. Michal Opas is a member of the Heart and Stroke/Richard Lewar Centre of Excellence. This work was supported by grants from the Canadian Institutes of Health Research and the Heart and Stroke Foundation of Ontario. The authors would like to express their thanks to Oxana Kolenchenko for providing the immunofluorescence images.

Simultaneously published in Berger J. (ed.): Advances in Cell and Molecular Biology. Kopp, České Budéjovice 2005, pp. 79-112.

\section{REFERENCES}

Bastianutto C., Clementi E., Codazzi F. et al.: Overexpression of calreticulin increases the
$\mathrm{Ca}^{2+}$ capacity of rapidly exchanging $\mathrm{Ca}^{2+}$ stores and reveals aspects of their lumenal microenvironment and function. J. Cell Biol. 130:847-855, 1995.

Basu S., Binder R.J., Ramalingam T., Srivastava P.K.: CD91 is a common receptor for heat shock proteins gp96, hsp90, hsp70, and calreticulin. Immunity 14:303-313, 2001.

Belcheva M.M., Clark A.L., Haas P.D. et al.: Mu and kappa opioid receptors activate ERK/MAPK via different protein kinase $\mathrm{C}$ isoforms and secondary messengers in astrocytes. J. Biol. Chem. 280:27662-27669, 2005.

Bouvard D., Molla A., Block M.R.: Calcium/calmodulin-dependent protein kinase II controls alpha5beta1 integrin-mediated inside-out signaling. J. Cell Sci. 111:657-665, 1998.

Brown M.C., Perotta J.A., Turner C.E.: Serine and threonine phosphorylation of the paxillin LIM domains regulates paxillin focal adhesion localization and cell adhesion to fibronectin. Mol. Biol. Cell 9:1803-1816, 1998.

Burridge K., Chrzanowska-Wodnicka M.: Focal adhesions, contractility, and signalling. Annu. Rev. Cell Dev. Biol. 12: 463-518, 1996.

Burridge K., Turner C.E., Romer L.H.: Tyrosine phosphorylation of paxillin and $\mathrm{pp} 125^{\mathrm{FAK}}$ accompanies cell adhesion to extracellular matrix: A role in cytoskeletal assembly. J. Cell Biol. 119:893-903, 1992.

Camacho P., Lechleiter J.D.: Calreticulin inhibits repetitive intracellular $\mathrm{Ca} 2+$ waves. Cell 82: 765-771, 1995.

Coppolino M.G., Woodside M.J., Demaurex N. et al.: Calreticulin is essential for integrinmediated calcium signalling and cell adhesion. Nature 386:843-847, 1997.

Coppolino M., Leung-Hagesteijn C., Dedhar S., Wilkins $\mathrm{J}$.: Inducable interaction of integrin $\alpha_{2} \beta_{1}$ with calreticulin-dependence on the activation state of the integrin. J. Biol. Chem. 270:23132-23138, 1995.

Crawford A.W., Michelson J.W., Beckerle M.C.: An interactionbetween zyxin and $\alpha$-actinin. J. Cell Biol. 116:1381-1393, 1992.

Dai E., Stewart M., Ritchier B. et al.: Calreticulin, a potential vascular regulatory protein, reduces intimal hyperplasia after arterial injury. Arterioscler. Thromb. Vasc. Biol. 17:23592368, 1997.

Darby P.J., Kwan C.Y., Daniel E.E.: Caveolae from canine airway smooth muscle contain the necessary components for a role in $\mathrm{Ca}(2+)$ handling. Am. J. Physiol Lung Cell Mol. Physiol. 279:L1226-L123, 2000.

Dedhar S.: Novel functions of calreticulin: interaction with integrins and modulation of 
gene expression. Trends Biochem. Sci. 19:269-271, 1994.

Eggleton P., Reid K.B.M., Kishore U., Sontheimer R.D.: Clinical relevance of calreticulin in systemic lupus erythematosus. Lupus 6:564571, 1997.

Fadel M.P., Dziak E., Lo C.M. et al.: Calreticulin affects focal contact-dependent but not close contact-dependent cell-substratum adhesion. J. Biol. Chem. 274:15085-15094, 1999.

Fadel M.P., Szewczenko-Pawlikowski M., Leclerc P. et al.: Calreticulin affects beta-catenin associated pathways. J. Biol. Chem. 276:27083-27089, 2001.

Fasolato C., Pizzo P., Pozzan T.: Delayed activation of the store-operated calcium current induced by calreticulin overexpression in RBL1 cells. Mol. Biol. Cell 9:1513-1522, 1998.

Ghiran I., Klickstein L.B., Nicholson-Weller A.: Calreticulin is at the surface of circulating neutrophils and uses CD59 as an adaptor molecule. J. Biol. Chem. 278:21024-21031, 2003.

Goicoechea S., Orr A.W., Pallero M.A. et al.: Thrombospondin mediates focal adhesion disassembly through interactions with cell surface calreticulin. J. Biol. Chem. 275:3635836368, 2000.

Goicoechea S., Pallero M.A., Eggleton P. et al.: The anti-adhesive activity of thrombospondin is mediated by the N-terminal domain of cell surface calreticulin. J. Biol. Chem. 277:3721937228, 2002.

Helenius A., Trombetta E.S., Hebert D.N., Simons J.M.: Calnexin, calreticulin and the folding of glycoproteins. Trends Cell Biol. 7:193-200, 1997.

Huang C., Jacobson K., Schaller M.D.: MAP kinases and cell migration. J. Cell Sci. 117:4619-4628, 2004.

Hutzfeld M.: The armadillo family of structural proteins. Int. Rev. Cytol. 186:179-224, 1999.

Ilic D., Damsky C.H., Yamamoto T.: Focal adhesion kinase: at the crossroads of signal transduction. J. Cell Sci. 110:401-407, 1997.

Illario M., Cavallo A.L., Monaco S. et al.: Fibronectin-induced proliferation in thyroid cells is mediated by alpha $\mathrm{v}$ beta 3 integrin through Ras/Raf-1/MEK/ERK and calcium/CaMKII signals. J. Clin. Endocrinol. Metab. 90:2865-2873, 2005.

Ishibe S., Joly D., Liu Z.X., Cantley L.G.: Paxillin serves as an ERK-regulated scaffold for coordinating FAK and Rac activation in epithelial morphogenesis. Mol. Cell. 16:257267, 2004.

Jouaville L.S., Ichas F., Holmuhamedov E.L. et al.: Synchronization of calcium waves by mitochondrial substrates in Xenopus laevis oocytes. Nature 377:438-441, 1999.
Juliano R.L., Haskill S.: Signal transduction from the extracellular matrix. J. Cell Biol. 120:57755, 1993.

Kornberg L., Juliano R.L.: Signal trasduction from the extracellular matrix: The integrin-tyrosine kinase connection. Trends Pharmacol. Sci. 13:93-95, 1992.

Kuwabara K., Pinsky D.J., Schmidt A.M. et al.: Calreticulin, an antithrombotic agent which binds to vitamin K-dependent coagulation factors, stimulates endothelial nitric oxide production, and limits thrombosis in canine coronary arteries. J. Biol. Chem. 270:81798187, 1995.

Liu N., Fine R.E., Simons E., Johnson R.J.: Decreasing calreticulin expression lowers the $\mathrm{Ca} 2+$ response to bradykinin and increases sensitivity to ionomycin in NG-108-15 cells. J. Biol. Chem. 269:28635-28639, 1994.

Lockwich T.P., Liu X., Singh B.B. et al.: Assembly of Trp1 in a signaling complex associated with caveolin-scaffolding lipid raft domains. J. Biol. Chem. 275: 11934-11942, 2000.

Lockwich T.P., Singh B.B., Liu X., Ambudkar I.S.: Stabilization of cortical actin induces internalization of Trp3-associated caveolar $\mathrm{Ca}^{2+}$ signaling complex and loss of $\mathrm{Ca}^{2+}$ influx without disruption of Trp3-IP3R association. J. Biol. Chem. 276:42401-42408, 2001.

McGreal E., Gasque P.: Structure-function studies of the receptors for complement $\mathrm{C} 1 \mathrm{q}$. Biochem. Soc. Trans. 30:1010-1014, 2001.

Meldolesi J., Pozzan T.: The heterogeneity of ER $\mathrm{Ca}^{2+}$ stores has a key role in nonmuscle cell signaling and function. J. Cell Biol. 142:13951398, 1998.

Mery L., Mesaeli N., Michalak M. et al.: Overexpression of calreticulin increases intracellular $\mathrm{Ca}^{2+}$-storage and decreases storeoperated $\mathrm{Ca}^{2+}$ influx. J. Biol. Chem. 271:93329339, 1996.

Michalak M., Burns K., Mesaeli N. et al.: Endoplasmic reticulum form of calreticulin modulates glucacorticoid-sensitive gene expression. J. Biol. Chem. 271:29436-26445, 1996.

Michalak M., Parker J.M.R., Opas M.: $\mathrm{Ca}^{2+}$ signaling and calcium binding chaperones of the endoplasmic reticulum. Cell Calcium 32:269-278, 2002.

Ogden C.A., deCathelineau A., Hoffmann P.R. et al.: $\mathrm{Clq}$ and mannose binding lectin engagement of cell surface calreticulin and cd91 initiates macropinocytosis and uptake of apoptotic cells. J. Exp. Med. 194:781-796, 2001.

Orr A.W., Pedraza E.C., Pallero M.A. et al.: Low density lipoprotein receptor-related protein is a calreticulin coreceptor that signals focal 
adhesion disassembly. J. Cell Biol. 161:11791189, 2003.

Orr A.W., Elzie C.A., Kucik F.D., Murphy-Ullrich J.E.: Thrombospondin signalling through the calreticulin/LDL receptor-related protein cocomplex stimulates random and directed cell migration. J. Cell Sci. 116:2917-2927, 2003.

Orr A.W., Pallero M.A., Murphy-Ullrich J.E.: Thrombospondin stimulates focal adhesion disassembly through Gi- and phosphoinositide 3-kinase-dependent ERK activation. J. Biol. Chem. 277:20453-20460, 2002.

Opas M., Szewczenko-Pawlikowski M., Jass G.K. et al.: Calreticulin modulates cell adhesiveness via regulation of vinculin expression. J. Cell Biol. 135:1913-1923, 1996.

Otey C.A.: pp $125^{\text {FAK }}$ in the focal adhesion. Int. Rev. Cytol. 167:161-183, 1996.

Papp S., Opas M.: Subcellular distribution of calreticulin. In Eggleton P., Michalak M. (eds.): Calreticulin. Landes Bioscience, Georgetown 2003, pp. 38-48.

Papp S., Dziak E., Michalak M., Opas M.: Is all of the endoplasmic reticulum created equal? The effect of the heterogeneous distribution of endoplasmic reticulum $\mathrm{Ca}^{2+}$-handling protein. J. Cell. Biol. 160:475-479, 2003.

Papp S., Fadel M., Opas M.: ER-to-cell surface signalling: calreticulin and cell adhesion. J. Appl. Biomed. 2:1-14, 2004.

Parodi A.J.: Role of N-oligosaccharide endoplasmic reticulum processing reactions in glycoprotein folding and degradation. Biochem. J. 348 Pt 1:1-13, 2000.

Petersen O.H., Tepikin A., Park M.K.: The endoplasmic reticulum: one continuous or several separate $\mathrm{Ca}^{2+}$ stores? Trends Neurosci. 24:271-276, 2001.

Pike S.E., Yao L., Setsuda J. et al.: Calreticulin and calreticulin fragments are endothelial cell inhibitors that suppress tumor growth. Blood 94:2461-2468, 1999.

Pike S.E., Yao L., Jones K.D. et al.: Vasostatin, a calreticulin fragment, inhibits angiogenesis and suppresses tumor growth. J. Exp. Med. 188:2349-2356, 1998.

Putney J.W., Jr.: “Kissin' cousins”: intimate plasma membrane-ER interactions underlie capacitative calcium entry. Cell 99:5-8, 1999.

Putney J.W. Jr.: Capacitative calcium entry: sensing the calcium stores. J. Cell Biol. 169:381-382, 2005.
Sacks D.B., Fujita-Yamaguchi Y., Gale R.D., McDonald J.M.: Tyrosine-specific phosphorylation of calmodulin by the insulin receptor kinase purified from human placenta. Biochem. J. 263:803-812, 1989.

Sastry S.K., Burridge K.: Focal adhesion: a nexus for intracellular signalling and cytoskeletal dynamics. Exp. Cell Res. 261:25-36, 2000.

Sugiyama T., Matsuda Y., Mikoshiba K.: Inositol 1,4,5-trisphosphate receptor associated with focal contact cytoskeletal proteins. FEBS Lett. 466:29-34, 2000.

Vandivier R.W., Ogden C.A., Fadok V.A. et al.: Role of surfactant proteins $\mathrm{A}, \mathrm{D}$, and $\mathrm{C} 1 \mathrm{q}$ in the clearance of apoptotic cells in vivo and in vitro: calreticulin and CD91 as a common collectin receptor complex. J. Immunol. 169:3978-3986, 2002.

Volonté D., Galbiati F., Pestell R.G., Lisanti M.P.: Cellular stress induces the tyrosine phosphorylation of caveolin-1 (Tyr14) via activation of $\mathrm{p} 38$ mitogen-activated protein kinase and c-Src kinase - evidence for caveolae, the actin cytoskeleton, and focal adhesions as mechanical sensors of osmotic stress. J. Biol. Chem. 276:8094-8103, 2001.

Wei Y., Yang X.W., Liu Q.M. et al.: A role for caveolin and the urokinase receptor in integrinmediated adhesion and signaling. J. Cell Biol. 144:1285-1294, 1999.

Wen Z., Guirland C., Ming G.L., Zheng J.Q.: A CaMKII/calcineurin switch controls the direction of $\mathrm{Ca}^{2+}$-dependent growth cone guidance. Neuron 43:835-846, 2004.

White T.K., Zhu Q., Tanzer M.L.: Cell surface calreticulin is a putative mannoside lectin which triggers mouse melanoma cell spreading. J. Biol. Chem. 270:15926-15929, 1995.

$\mathrm{Xu}$ W., Longo F.J., Wintermantel M.R. et al.: Calreticulin modulates capacitative $\mathrm{Ca}^{2+}$ influx by controlling the extent of inositol 1,4,5trisphosphate-induced $\mathrm{Ca}^{2+}$ store depletion. J. Biol. Chem. 275:36676-36682, 2000.

Zhu Q., Zelinka P., White T., Tanzer M.L.: Calreticulin-integrin bidirectional signaling complex. Biochem. Biophys. Res. Commun. 232:354-358, 1997. 